\title{
Evaluación del desempeño ambiental basado en la norma técnica ISO 14001:2004 con el fin de determinar estrategias para su implementación en la Universidad de Cundinamarca*
}

Environmental performance assessment based on the technical standard Environmental management systems ISO 14001in order to determine strategies for its implementation at the

University of Cundinamarca

Ricardo Andrés Jimenes Nieto***

Universidad de Cundinamarca

Sneyther Nuñez Cruz*****

Alcaldía Municipal de Tocancipá

RESUMEN

\footnotetext{
* Artículo de Investigación.

** Sneyther Nuñez Cruz. Administrador de Empresas. Magister en Calidad y Gestión Integral Profesional Especializado de la Alcaldía Municipal de Tocancipá. Correo electrónico: sneyther@hotmail.com

*** Ricardo Andrés Jimenez Nieto. Ingeniero Electrónico. Especialista en Instrumentación Electrónica. Magister en Calidad y Gestión Integral. Director de Sistemas y Tecnología Universidad de Cundinamarca. Correo electrónico: r_a_jimenez@hotmail.com
} 
Esta investigación lleva a cabo el diagnóstico del desempeño ambiental de la Universidad de Cundinamarca en su sede principal ubicada en la ciudad de Fusagasugá, con el fin de analizar los beneficios de aplicar un sistema de gestión ambiental y construir estrategias para su implementación. La investigación se enmarca en los conceptos de desarrollo sostenible y gestión ambiental así como en la problemática ambiental causada por las universidades. Como resultado se evidencia la afectación directa del medio ambiente y se concluye que es necesario aplicar una herramienta que facilite a la alta dirección mejorar el desempeño de las actividades administrativas, académicas y operativas para no continuar impactando el medio ambiente; también se determinan los beneficios y las ventajas que pueden aprovecharse en la implementación de un sistema de gestión ambiental a partir de los sistemas de gestión existentes, como el sistema de gestión de la calidad basado en la norma técnica NTCGP 1000 y el sistema de gestión del control basado en el modelo MECI.

Palabras clave: evaluación del desempeño ambiental, ISO 14001, gestión ambiental, revisión ambiental inicial (RAI), desarrollo sostenible.

\section{ABSTRACT}

This research carried out the environmental performance diagnosis at the University of Cundinamarca in its headquarters located in Fusagasuga city, in order to analyze the benefits of implementing an environmental management system and build strategies for its implementation. The research is part of the concepts of sustainable development and environmental management as well as environmental problems caused by the Universities. As a result the direct impact over the environment is evidenced and it is concluded that it is necessary to apply a tool providing senior management to improve the performance of administrative, academic and non-operating activities to not continue impacting the environment; also determine the benefits and advantages that can be exploited in the implementation of an environmental management system based on the existing management systems such as quality management system based on the technical standard NTC GP 1000 and the management control system based on the MECI model.

Keywords: Environmental performance assessment, ISO 14001, environmental management, initial environmental review (IAR), sustainable development.

\section{INTRODUCCIÓN}

Guillermo Guerra (2000) explica que las organizaciones en las que confluyen diariamente gran cantidad de personas y utilizan abundante cantidad de recursos para el desarrollo de las actividades que les permiten cumplir con sus objetivos misionales presentan grandes riesgos de afectar el medio ambiente y por tanto, de acuerdo con López (2003), la calidad de vida de sus funcionarios, clientes internos, usuarios de sus servicios, así como de la comunidad del entorno en que adelantan sus actividades y de la sociedad en general. Según Fuente (1998) y Alfie (2006), tal es el caso de los centros de educación superior, que además de contar con una buena afluencia de participantes en diferentes horarios, utilizan en sus procesos de gestión de recursos educativos (apoyo académico), elementos que por su composición ponen en riesgo el medio ambiente con las consecuencias que de ello se derivan. La Universidad de Cundinamarca busca identificar aquellas actividades desarrolladas al interior de su sede principal que generan algún impacto significativo sobre el medio ambiente y, teniendo 
en cuenta que los sistemas de gestión ambiental son ampliamente utilizados por las organizaciones, determinar la brecha existente entre su desempeño actual y los requisitos de la norma técnica ISO 14001:2004 (ICONTEC, 2004). Pensando en el rechazo al cambio, analizar las ventajas de una posible implementación permitirá a la institución visualizar los beneficios financieros, sociales, legales, ambientales y de imagen. Finalmente el desarrollo de este proyecto dará a la Universidad de Cundinamarca unas estrategias para integrarse al actual Sistema Integrado de Gestión y Control -SIGC ofreciendo una plataforma de gestión para su implementación.

Como hipótesis de la investigación se planteó que la correlación entre los requisitos del actual Sistema Integrado de Gestión y Control -SIGG y los lineamientos de acreditación exigidos por el Consejo Nacional de Acreditación de Colombia - CNA, fija las políticas necesarias para que la actuación de los procesos que se desarrollan en la Universidad de Cundinamarca Sede Central no atenten contra el medio ambiente.

El desarrollo de la investigación se estructuro en tres objetivos, el primero consiste en elaborar un diagnóstico que identifique los aspectos e impactos ambientales derivados de las actividades desarrolladas en la Universidad de Cundinamarca en su sede principal mediante la aplicación de una Revisión Ambiental Inicial. El segundo, en analizar los beneficios de un Sistema de Gestión Ambiental en la Universidad de Cundinamarca. Finalmente, construir estrategias para la implementación de un Sistema de Gestión Ambiental para la Universidad de Cundinamarca sede Fusagasugá.

Algunos antecedentes permiten contextualizar el reto de las Instituciones de Educación Superior de educar para el desarrollo sustentable y de implementar Sistemas de Gestión Ambiental, como es el caso de algunas Instituciones de Educación Superior como la Universidad Politécnica de Madrid, la Universidad Nacional de Costa Rica y la Universidad Tecnológica de Chile y en Colombia las Universidades Cooperativa de Colombia y Surcolombiana; así mismo, es notorio el caso de la Universidad de Harvard en Cambridge, Massachusetts - Estados Unidos, que comprometida con el desarrollo sostenible creo su Green Campus, y viene trabajando en diferentes temas como la construcción verde (Green Building) para renovar y construir edificios más sostenibles y eficientes con la energía, para lo cual ha adoptado amplias normas de construcción verde en la gestión de sus proyectos implementando distintos niveles de diseño integrado, ciclo de vigencia del costo, modelamiento de energía, y diversas métricas de desempeño ambiental, tales como el reciclaje de residuos de la construcción, conservación del agua y la educación de sus ocupantes, lo que le ha hecho acreedora de la certificación LEDD 1 .

\section{METODOLOGÍA}

Se conformaron grupos especializados en cada componente relevante para la gestión ambiental: Residuos, Agua, Energía y Perceptual. Debido a que la Universidad no cuenta con un procedimiento para la identificación de sus aspectos e impactos ambientales, colectivamente se construyó la siguiente estructura como método de recolección, clasificación y selección de información (Bonilla y Rodríguez, 2005) (Figura 1):

1 Acrónimo de Leadership in Energy Æ̈ Environmental Design, sistema de certificación de edificios sostenibles, desarrollado por el Consejo de la Construcción Verde de Estados Unidos (US Green Building Council). 
Figura 1. Estructura del diagnóstico ambiental
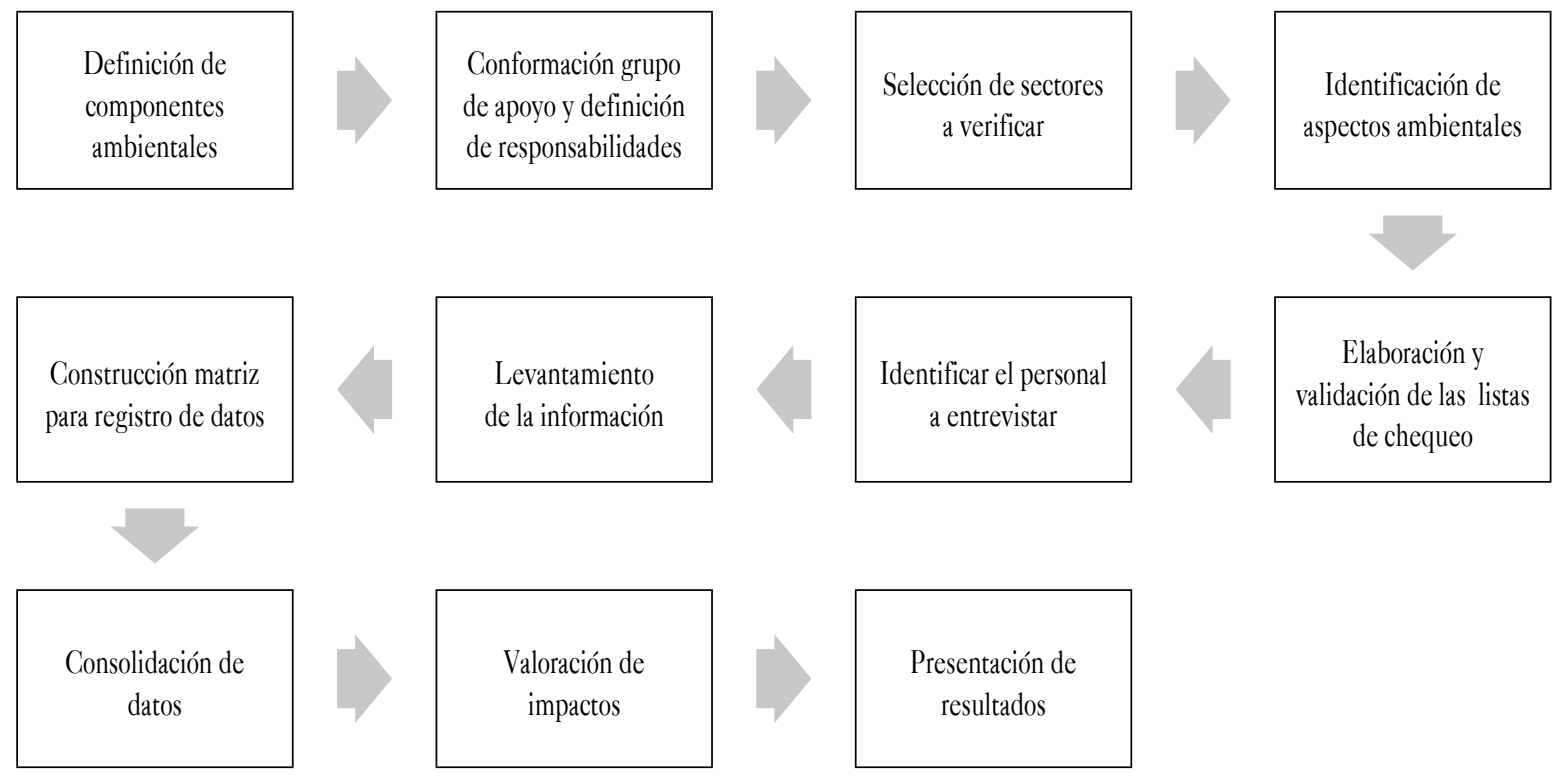

Elaboración y validación de las listas de chequeo

Fuente: equipo SGA Universidad de Cundinamarca.

Los aspectos ambientales identificados para la Universidad fueron seleccionados de la codificación de aspectos e impactos ambientales descritos en el módulo de Gestión Ambiental orientado por la Ingeniera Magda González Rodríguez en la Maestría en Calidad y Gestión Integral de la Universidad Santo Tomás, tales aspectos identificados son propios a la actividad de la Universidad de Cundinamarca y se relacionan a continuación (Figura 2):

Figura 2. Aspectos ambientales Universidad de Cundinamarca

\begin{tabular}{|c|c|c|}
\hline Aspecto Ambiental & Impacto Ambiental & Tipo \\
\hline \multicolumn{3}{|c|}{ Consumo de Energía e Insumos } \\
\hline Consumo de energía & $\begin{array}{l}\text { Disminución de recursos naturales } \\
\text { Aporte al calentamiento global }\end{array}$ & - \\
\hline $\begin{array}{l}\text { Consumo de insumos: ej.: papel y otros insumos de oficina, } \\
\text { detergentes, productos químicos, combustibles }\end{array}$ & Aumento en la demanda de recursos & - \\
\hline \multicolumn{3}{|c|}{ Agua } \\
\hline Consumo de agua & Disminución del recurso hídrico & - \\
\hline Aprovechamiento de aguas lluvia & Disminución de la presión sobre el recurso hídrico & + \\
\hline Generación de aguas residuales (domésticas o industriales) & Incremento del caudal de aguas residuales a ser tratadas & - \\
\hline Vertimiento de Aguas Residuales Domésticas & Contaminación del Agua & - \\
\hline
\end{tabular}


Evaluación del desempeño ambiental basado en la norma técnica ISO 14001:2004 con el fin de determinar estrategias para su...

\begin{tabular}{|c|c|c|}
\hline Aspecto ambiental & Impacto ambiental & Tipo \\
\hline Vertimiento de Aguas Residuales Industriales & Contaminación del Agua & - \\
\hline Recirculación Agua & Aprovechamiento del Recurso & + \\
\hline \multicolumn{3}{|c|}{ Residuos } \\
\hline Generación Residuos Peligrosos & $\begin{array}{l}\text { Aumento de Residuos Peligrosos a disponer } \\
\text { Contaminación del aire }\end{array}$ & - \\
\hline Generación Residuos No Peligrosos & $\begin{array}{l}\text { Aumento de Residuos No peligrosos a disponer } \\
\text { Presión sobre el relleno sanitario }\end{array}$ & - \\
\hline $\begin{array}{l}\text { Aprovechamiento de Residuos (Reciclaje, Reutilización, } \\
\text { Reducción, comercialización, etc.) }\end{array}$ & Disminución de la Cantidad de Residuos a disponer & + \\
\hline \multicolumn{3}{|c|}{ Suelo } \\
\hline Uso del suelo & $\begin{array}{l}\text { Cambio en el uso del suelo } \\
\text { Alteración de las características del suelo }\end{array}$ & - \\
\hline Derrames de sustancias (Combustibles, Químicos, etc.) & $\begin{array}{l}\text { Contaminación del Suelo o Agua } \\
\text { Aumento de la cantidad de Residuos peligrosos a disponer }\end{array}$ & - \\
\hline Aplicación de agroquímicos & Alteración de las características del suelo & - \\
\hline Uso de abonos orgánicos y controles biológicos & Aporte de nutrientes, recuperación y mejora del suelo & + \\
\hline \multicolumn{3}{|c|}{ Fauna y Flora } \\
\hline Consumo de madera & Disminución del recurso forestal & - \\
\hline Interacción con fauna & $\begin{array}{l}\text { Desplazamiento de especies } \\
\text { Variación de procesos migratorios } \\
\text { Alteraciones en el comportamiento de especies silvestres } \\
\text { Pérdida de biodiversidad }\end{array}$ & - \\
\hline Deforestación, poda o tala de árboles & $\begin{array}{l}\text { Disminución del recurso forestal } \\
\text { Pérdida de biodiversidad } \\
\text { Degradación de suelos }\end{array}$ & - \\
\hline Reforestación & $\begin{array}{l}\text { Recuperación del recurso forestal } \\
\text { Mitigación de cambio climático }\end{array}$ & + \\
\hline Descarga de agentes contaminantes en agua o suelo & Afectación de flora y fauna & - \\
\hline \multicolumn{3}{|c|}{ Visual y Otros } \\
\hline Uso de publicidad exterior visual & $\begin{array}{l}\text { Contaminación Visual } \\
\text { Alteración del paisaje }\end{array}$ & - \\
\hline Falta de orden y aseo en áreas exteriores & Contaminación Visual & - \\
\hline Mantenimiento del Paisaje y Urbanismo & Mejoramiento Visual & + \\
\hline Ocupación de espacio público & Afectación a la Comunidad & - \\
\hline Generación de empleo & Aporte al desarrollo de la comunidad & + \\
\hline
\end{tabular}

Fuente: curso Gestión Ambiental Ing. Magda González Rodríguez. 
Como herramienta estandarizada para la identificación de los aspectos, impactos y riesgos ambientales se construyeron listas de verificación guía basadas en la Guía Técnica Colombiana GTC 93 (ICONTEC, 2003), convirtiéndose este ejercicio en la Revisión Ambiental Inicial RAI; para su desarrollo, fue necesario dividir el campus universitario en tres sectores, la granja se consideró como un cuarto sector. Las listas de verificación fueron aplicadas mediante entrevistas realizadas a los responsables de cada área de la Universidad y la apreciación de los entrevistadores a través de la observación. Posteriormente se empleó el método de Análisis de Diferencias (Gap Analysis) mediante el diseño y aplicación de un instrumento de verificación que permite determinar el estado actual de la Universidad frente al cumplimiento de los requisitos de la NTC ISO 14001:2004; se incluye una revisión de los requisitos legales ambientales aplicables en la Universidad (Patiño, 1999).

Posteriormente, conforme a Conesa (1993), se procesa toda la información recolectada aplicando la tabulación de los datos, el análisis de los mismos y aplicando metodologías propias de la evaluación de impactos y riesgos ambientales, lo que permite determinar cuáles impactos ambientales se pueden consideran como críticos, severos, moderados o insignificantes; también se determina qué riesgos ambientales son catastróficos, mayores, moderados, menores e insignificantes. De igual forma se calculó el consumo en los componentes Agua y Energía; y se clasificó el tipo de residuo mediante el cuarteo en el componente Residuos.

Por último, se presenta el informe final que establece la conveniencia de implementar el Sistema de Gestión Ambiental SGA con el que se busca eliminar los impactos ambientales negativos que genera la Universidad de Cundinamarca, sede Fusagasugá, y las estrategias para incorporarlo como nuevo modelo al actual Sistema Integrado de Gestión y Control SIGC (Abril, Enríquez y Sánchez, 2006).

\section{RESULTADOS Y DISCUSIÓN}

En el ejercicio de identificación de aspectos, riesgos e impactos ambientales en cada sector de la Universidad de Cundinamarca, se determina que la Universidad produce residuos como papel, cartón, vidrio y el plástico que conforman más del $50 \%$ de la producción de residuos. Otros residuos como el icopor, los biosanitarios, ordinarios y orgánicos completan la composición física de los residuos.

En el componente Residuos, los impactos ambientales son significativos al punto de ser considerados como críticos, ya que se presenta contaminación por derrames químicos, físicos, microorganismos patógenos o elementos radioactivos, la generación de vectores, el peligro de contagiarse de enfermedades graves, la destrucción de recursos naturales y la acumulación de residuos peligrosos.

En el componente Agua se determina un impacto ambiental alto, la degradación de la calidad del agua y otros impactos considerados medianos como la pérdida de agua por evaporación, el consumo desmedido y por ende la generación de altos costos en la facturación de este servicio. Como complemento al ejercicio de diagnóstico del componente Agua, se tomó en el número de sanitarios defectuosos considerados como no aceptables, su respectiva capacidad de descarga en metros cúbicos, la cantidad de usuarios (población de Fusagasugá), el costo del metro cubico $\left(\mathrm{m}^{3}\right)$ y se comparó con la capacidad de descarga de una cisterna ahorrativa, obteniéndose los siguientes resultados que demuestran el consumo desmedido evidenciado en la valoración de impactos (Tabla 1): 
Tabla 1. Consumo agua U. de Cundinamarca

\begin{tabular}{|l|c|r|r|}
\hline \multicolumn{2}{|l|}{ Total sanitarios no aceptables } & 40 \\
\hline \multicolumn{2}{|l|}{ Total usuarios Universidad } & 5061 \\
\hline \multicolumn{2}{|l|}{ Valor metro cubico $\left(\mathrm{m}^{3}\right)$} & $\$ 920$ \\
\hline $\begin{array}{c}\text { Tipo de } \\
\text { Cisterna }\end{array}$ & $\begin{array}{c}\text { Capacidad } \\
\left(\mathrm{m}^{3}\right)\end{array}$ & $\begin{array}{c}\text { Consumo de una } \\
\text { descarga al día }\end{array}$ & Costo $(\$)$ \\
\hline $\begin{array}{l}\text { No } \\
\text { aceptables }\end{array}$ & 0,012 & 2.429 & 2.234 .938 \\
\hline Ahorrativas & 0,006 & 1.215 & 1.117 .469 \\
\hline
\end{tabular}

Fuente: elaboración propia.

En cuanto al componente Energía se determinó que el principal impacto es el agotamiento del recurso por el consumo desmedido. Consumo observado en la facturación del último año y ocasionado por el deterioro de las instalaciones eléctricas que presenta la institución. De igual forma, no se evidenció el uso de ningún tipo de tecnología para ahorrar este recurso ni el cumplimiento de las normativas pertinentes.

En el componente Perceptual se detectaron impactos significativos como el deterioro del paisaje y los cambios en la composición del suelo en algunos sectores de la institución.

Como parte integral del diagnóstico ambiental se realizó el análisis de diferencias (GAP) entre el desempeño actual de la Universidad frente a los requisitos de la norma ISO 14001, encontrándose que la Universidad posee un avance del once por ciento (11\%) en la implementación de un sistema de gestión ambiental; este valor se logra debido a la existencia de algunos elementos dispuestos en los actuales sistema de gestión de la calidad y del control integrados en el SIGC. El avance de ISO 14001 por cada requisito, se puede observar en la Figura 3.

Figura 3. Cumplimiento de la norma técnica ISO 14001:2005 en la Universidad de Cundinamarca

\section{$\%$ de cumplimiento}

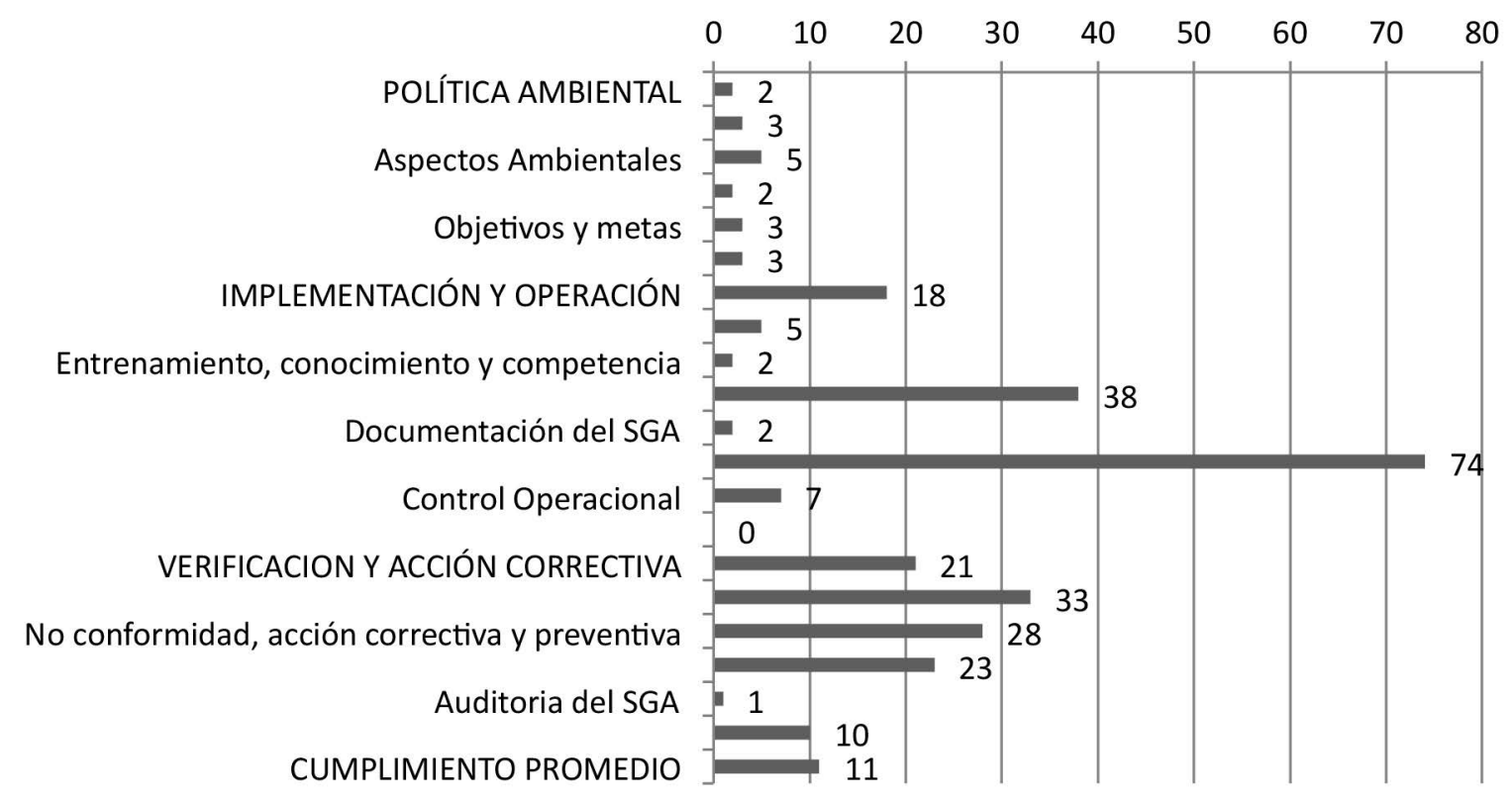

Fuente: elaboración propia. 
El resultado del diagnóstico lleva al segundo objetivo que corresponde al análisis de los beneficios de implementar un SGA en la Universidad de Cundinamarca, sede Fusagasugá; al respecto se puede establecer que la Universidad obtendría un alto beneficio en los aspectos, financiero, ambiental, social, de imagen y legal, los cuales fueron agrupados como lo muestra la Tabla 2:

Tabla 2. Beneficios de implementar el SGA en la U. de Cundinamarca

\begin{tabular}{|c|c|c|}
\hline No. & CONTEXTO & BENEFICIO \\
\hline \multirow{6}{*}{1} & \multirow{6}{*}{ Financiero } & $\begin{array}{l}\text { Reducción de Costos. En la medida en que se implemente un SGA en la UDEC, se verá reflejado el ahorro, } \\
\text { representado en; Consumo de Energía, reducción de residuos y emisiones, aprovechamiento mediante reciclaje y, } \\
\text { sanciones por incumplimiento a la normatividad ambiental. }\end{array}$ \\
\hline & & $\begin{array}{l}\text { Facilita el trabajo de los responsables de cada área. El sistema documentado permite establecer controles que } \\
\text { conllevan a mejorar los procesos. }\end{array}$ \\
\hline & & $\begin{array}{l}\text { Mejoramiento de proveedores. Las relaciones de compra de insumos, proveedores que cumplan con exigencias } \\
\text { mínimas de seguridad ambiental, establecidas por la UDEC, convirtiéndose en relaciones mutuamente } \\
\text { beneficiosas. }\end{array}$ \\
\hline & & $\begin{array}{l}\text { Limitación del riesgo. Al momento en que se implemente el SGA en la UDEC, es claro que habrá una serie de } \\
\text { esfuerzos para el cumplimiento de los objetivos y normatividad relacionados que evitarán casos de sanciones } \\
\text { ambientales. }\end{array}$ \\
\hline & & La implementación de un SGA en la UDEC atraería nuevos clientes que representaría mayores ingresos. \\
\hline & & Reduce el consumo de energía y las emisiones contaminantes. \\
\hline \multirow{9}{*}{2} & \multirow{9}{*}{ Ambiental } & Compromete a toda la sociedad al cuidado de los recursos naturales. \\
\hline & & Previene y Controla el impacto ambiental de sus actividades y servicios. \\
\hline & & Poder acceder a mayores recursos públicos, encaminados a la protección del medio ambiente. \\
\hline & & Disminuye costos al minimizar los desechos y las emisiones. \\
\hline & & $\begin{array}{l}\text { Adquirir una cultura Ambiental representa una gran contribución y un gran compromiso a la comunidad en general, } \\
\text { mediante los programas de sensibilización que implemente la Universidad. }\end{array}$ \\
\hline & & $\begin{array}{l}\text { Transferencia de Tecnologías. Al implementarse un SGA, la Universidad puede desarrollar tecnologías que } \\
\text { permiten disponer de procesos más limpios y eficientes. }\end{array}$ \\
\hline & & Garantiza un mejor compromiso con el medio ambiente. \\
\hline & & $\begin{array}{l}\text { Identifica los impactos ambientales lo que conlleva a la aplicación oportuna de los controles necesarios para su } \\
\text { mitigación. }\end{array}$ \\
\hline & & Reduce los riesgos ambientales. \\
\hline \multirow{3}{*}{3} & \multirow{3}{*}{ Social } & $\begin{array}{l}\text { Garantiza una mejor calidad de vida para su entorno (Comunidad estudiantil y Entorno del a Universidad), al } \\
\text { reducir los impactos ambientales que genera la actividad desarrollada en la Universidad. }\end{array}$ \\
\hline & & Establece mejores relaciones con la comunidad. \\
\hline & & $\begin{array}{l}\text { Ser más eficiente, por el establecimiento de procesos ambientales que dinamiza y articula todas las áreas de la } \\
\text { Universidad. }\end{array}$ \\
\hline
\end{tabular}




\begin{tabular}{|c|c|c|}
\hline No. & CONTEXTO & BENEFICIO \\
\hline \multirow{5}{*}{3} & \multirow{5}{*}{ Social } & Establecería comuniaciones y relaciones con las partes interesadas, Internas y Externas. \\
\hline & & $\begin{array}{l}\text { Identificación de los impactos ambientales y por lo tanto la aplicación a tiempo de los controles necesarios para su } \\
\text { mitigación. }\end{array}$ \\
\hline & & $\begin{array}{l}\text { Mejora en la calidad del servicio. Por cuanto el diseño de un proceso documentado, establece la metodología a } \\
\text { seguir para su desempeño que ayuda a reducir los impactos ambientales, que repercute positivamente en el medio } \\
\text { ambiente. }\end{array}$ \\
\hline & & $\begin{array}{l}\text { Ayuda a contar con una estructura en programas de capacitación y entrenamiento acorde con los posibles impactos } \\
\text { ambientales identificados. }\end{array}$ \\
\hline & & $\begin{array}{l}\text { Incrementa los niveles de seguridad. Al implementarse un SGA en la UDEC, obliga a cumplir una serie de } \\
\text { procedimientos para el almacenamiento y tratamiento de productos peligrosos, con lo que se genera una } \\
\text { tranquilidad y seguridad en el entorno. }\end{array}$ \\
\hline \multirow{9}{*}{4} & \multirow{9}{*}{ Imagen } & $\begin{array}{l}\text { Conformidad con las exigencias de la población estudiantil y la comunidad (Clientes Internos y externos). Cada vez } \\
\text { es más la preocupación de la comunidad por el tema ambiental, sin importar su formación, así su conocimiento sobre } \\
\text { normatividad ambiental sea escaso, ésta advierte sobre las acciones que se generan y afectan su entorno; más aún la } \\
\text { población educativa, la Universidad es el templo del conocimiento y la mejor manera de educar es con el ejemplo. }\end{array}$ \\
\hline & & $\begin{array}{l}\text { Ventaja competitiva, por cuanto se destacaría dentro del sector universitario; más aún por tratarse de una Institución } \\
\text { Pública. }\end{array}$ \\
\hline & & Mejora de la Imagen Institucional y permite la mejora continua. \\
\hline & & $\begin{array}{l}\text { Mejora la comunicación entre las diferentes áreas. Por tratarse de un sistema de gestión documentado, se articulan } \\
\text { las acciones estableciendo responsabilidades, que ayudan a cumplir con las funciones asociadas a su puesto de } \\
\text { trabajo. }\end{array}$ \\
\hline & & $\begin{array}{l}\text { Capacidad para cumplir con expectativas internacionales. Aunque en la actualidad la implementación de un SGA } \\
\text { es voluntario, la preocupación por el tema ambiental es mundial, y en poco tiempo, será necesidad impuesta por la } \\
\text { sociedad en general. }\end{array}$ \\
\hline & & Genera disciplina organizacional. \\
\hline & & $\begin{array}{l}\text { El implementación de la norma ISO } 14001 \text { es un elemento diferenciador en la UDEC con respecto a otras } \\
\text { Universdides del sector Público, que lograría atraer un buen porcentaje de estudiantes. }\end{array}$ \\
\hline & & Asegura el compromiso entre la Alta dirección con todo el personal. \\
\hline & & $\begin{array}{l}\text { El compromiso que se adquiere al implementar el SGA, hace que la Universidad sea reconocida en el ámbito } \\
\text { mundial. }\end{array}$ \\
\hline \multirow{5}{*}{5} & \multirow{5}{*}{ Legal } & Conformidad con la legislación ambiental vigente en el país. \\
\hline & & Inspiraría confianza y fidelidad en actuales y potenciales estudiantes. \\
\hline & & Articulación con los tratados internacionales en materia ambiental. \\
\hline & & Facilita la obtención de permisos y licencias por pasar a ser una organización autocontrolada. \\
\hline & & $\begin{array}{l}\text { Consistencia de políticas. Por tratarse de políticas establecidas por el Rector de la Universidad (alta dirección), } \\
\text { obliga al compromiso de cumplimiento por parte de los funcionarios y de la comunidad estudiantil, que conlleva a } \\
\text { un mutuo incremento de confianza. }\end{array}$ \\
\hline
\end{tabular}

Fuente: elaboración propia. 
De acuerdo con los resultados del diagnóstico ambiental, la Universidad de Cundinamarca impacta significativamente con el desarrollo de sus actividades cotidianas en cuatro importantes componentes del medio ambiente (agua, energía, residuos y perceptual); sin embargo, la gestión que realiza sobre estos componente no supera el $11 \%$ al tomar como referente los requisitos mínimos que establece la norma ISO14001:2004; y como lo explican Cascio, Woodside y Mitchell (1997), conforme a la base teórica de la presente investigación, la implementación de un SGA debe formar parte de la Responsabilidad Social, que le permitiría a la Universidad manifestar su compromiso por mantener una relación estable entre el sistema social y el sistema llamado Tierra.

Para desarrollar este compromiso se propone la implementación del SGC mediante las siguientes estrategias:

Una de las estrategias de implementación consiste en crear una ventaja competitiva sobre otras Universidades ubicadas en la misma Región a partir de un modelo operativo consciente del medio ambiente que consolide una imagen Institucional a la altura de sus pares internacionales, lo que consecuentemente atraerá proyectos de inversión afines al portafolio de servicios académicos y profesionales patrocinados por aquellas empresas que ya operan con políticas ambientales. Esa ventaja competitiva derivará del conocimiento, las capacidades y las relaciones intangibles que se pueden desarrollar con los funcionarios de la Institución.

Otra estrategia consiste en hacer de los modelos de gestión herramientas que permitan operar la Universidad de Cundinamarca efica: y eficientemente dando cumplimiento a las expectativas del MEN de acuerdo a los requisitos minimos y los lineamientos de alta calidad. Para que esto sea posible es necesario que la Alta Dirección traslade esta estrategia al nivel operativo, primero, alineando todas las Directrices (Proyecto Educativo Institucional,
Plan de Desarrollo, Políticas de la Calidad, Ambiental y del Control, entre otros) y luego enfocando toda la Universidad a trabajar por el logro de los mismos objetivos. La Alta Dirección debe concientizarse que para alcanzar los objetivos necesita que todos sus funcionarios trabajen enfocados y alineados a estas directrices, por consiguiente debe llevarse esta estrategia a un nivel muy operativo de tal forma que cada funcionario ligado a un determinado proceso de gestión aporte con su trabajo a la eficacia y eficiencia institucional esperada.

Como el actual Sistema Integrado de Gestión y Control de la Universidad de Cundinamarca está conformado por el Sistema de Gestión de la Calidad basado en la NTCGP 1000:2009 y el Sistema de Gestión del Control basado en el MECI: 2005, la implementación del Sistema de Gestión Ambiental tendrá un desarrollo favorable por tratarse de un modelo basado en estándares ISO con estructuras similares.

La primera actividad de esta estrategia de integración para facilitar la implementación del SGA es revisar el nivel de madurez de cada uno de los sistemas de gestión antes mencionados.

Otra estrategia de integración consiste en crear una plataforma de gestión que permita optimizar los recursos, los costos y los esfuerwos dispuestos para el desarrollo de las políticas y objetivos ambientales y de la calidad, evitando duplicidad de actividades que tienen como fin el mismo propósito en la gestión. De esta forma, el objetivo de la estrategia es aportar para que la integración del SGA al actual Sistema Integrado de Gestión y Control incluya en sus procesos los criterios y las especificaciones necesarias para lograr la satisfacción de todas las partes interesadas (usuarios y comunidades ambientales) de forma simultánea y comprometida con la mejora continua. 
Si la Universidad desea iniciar el proceso de implementación, se advierte la importancia absoluta de contar con el total compromiso de la Alta Dirección pues del liderazgo que tenga dependerá que toda la Institución se movilice para que el nuevo sistema integrado de gestión sea efectivo. Por lo anterior se propone:

- Realizar una valoración de los alcances de cada sistema de gestión para determinar los procesos comunes.

- Realizar un análisis detallado de las expectativas de los usuarios que se haya comprometido la Universidad a satisfacer de acuerdo a su capacidad, a su planeación estratégica, y a los lineamientos del Consejo Nacional de Acreditación, CNA ligados con el mismo. Lo anterior con el fin de determinar los requisitos que están relacionados con los aspectos ambientales con los que la organización se comprometa en sus objetivos ambientales.

- Definir si se desea tener una Política Integrada de Gestión o si se conservan las políticas por separado.

- Establecer controles operativos en aquellas actividades incluidas tanto en los procesos misionales (cadena de valor) como los estratégicos, de apoyo y de evaluación y seguimiento para mitigar/eliminar los impactos ambientales y garantizar que las características de calidad del producto académico sean cumplidas.

- Crear una sola estructura a los procesos que resulten comunes a los dos tipos de gestión, ej.: control de documentos, registros, comunicación interna. En el caso del seguimiento y la medición se pueden conformar áreas integradas con las dos competencias de forma tal que se puedan hacer auditorías integradas de gestión.
- Incluir los dos aspectos: calidad y ambiental en la revisión por la alta dirección con el fin de ahorrar costos y examinar el desempeño de la organización con una mirada sistémica.

- Incorporar el tema medioambiental en las diferentes cátedras impartidas en los planes de estudio con el fin de crear conciencia en sus estudiantes, docentes y administrativos.

- Educar con el ejemplo, mostrar el verdadero compromiso por el medio ambiente implementando el sistema de gestión ambiental de forma tal que la preocupación ambiental no solo sea una exigencia impartida en los salones.

\section{CONCLUSIONES}

El resultado que arroja el diagnóstico realizado a la Universidad de Cundinamarca, sede Fusagasugá, deja en claro la necesidad de implementar un modelo de gestión ambiental en la Institución, ya que se generan grandes impactos y su desempeño ambiental es pobre, dado que el cumplimiento de los aspectos ambientales es muy bajo.

Aunque se evidencia algunos compromisos de la alta dirección, como la aprobación de esta investigación y la creación de un equipo interdisciplinario de apoyo a la misma, la asignación de recursos económicos y el establecimiento de un espacio de trabajo provisional, esta debe manifestar la decisión de apoyar y propiciar los mecanismos necesarios para poder desarrollar las acciones necesarias para lograr la implementación del SGA, herramienta con la que se garantiza el cumplimiento de metas ambientales y económicas, y que a su vez se integren con el actual SIGC con que cuenta la Universidad de Cundinamarca. Lo anterior, si se tiene 
en cuenta que la Visión, el PEI y el Plan rectoral de la Universidad no contemplan el componente ambiental.

La implementación del SGA en la Universidad de Cundinamarca generaría beneficios, por cuanto los costos que representa la implementación del SGA se recuperarían en corto plazo; además, obtendría fortalezas en los aspectos financiero, ambiental, social, imagen y social.

Se plantearon estrategias para la implementación del SGC, que de ser acogidas por la Universidad de Cundinamarca orientarán la ruta de aplicación y podrán agilizar este proceso. La Institución debe hacerse la siguiente pregunta: ¿Estamos en capacidad de ejecutar estas estrategias? Toda vez que no solo es necesario contar con una buena estrategia, pues ejecutarla correctamente definirá la eficacia y efectividad de la misma.

\section{REFERENCIAS}

Abril, C., Enríquez, A. y Sánchez, J. (2006). Manual para la integración de sistemas de gestión de Calidad, Medio Ambiente y Prevención de Riesgos Laborales. Madrid. Edita FUNDACION CONFOMETAL.

Alfie, M. (2006). Retos y Desafíos en la Universidad Autónoma Metropolitana-Azcapotzalco. México D.F.: Revista El Cotidiano.

Bonilla, E. y Rodríguez, P. (2005). Más allá del dilema de los métodos. La investigación en ciencias sociales. En: Métodos cuantitativos y cualitativos. 3 ed. Bogotá D.C: Editorial Norma.

Cascio, J., Woodside, G. y Mitchell, P. (1997). Guía ISO 14000. Las nuevas normas internacionales para la administración ambiental. México: McGraw Hill.
Conesa, V. (1993). Guía metodológica para la evaluación del impacto ambiental. 2 ed. Madrid: Editorial Mundi-Prensa.

Fuente, M. (1998). La Dimensión Ambiental en la formación Profesional. Una mirada sobre la pertinencia social de la Universidad pública. Profesor Investigador Universidad del Mar. México. Revista Dimensión. Recuperado de http://www.umar. $\mathrm{mx} /$ revistas/6/dimension.pdf

Guerra, G. (2000). Ética y responsabilidad Social del Agronegocio en un mundo globalizado. En: Revista Mexicana de Agronegocios. Sociedad Mexicana de Administración Agropecuaria A.C., Universidad Autónoma de la Laguna y La Universidad Autónoma Agraria "Antonio Narro". Recuperado de http://www.redalyc.org/pdf/141/14106411.pdf

Instituto Colombiano de Normas Técnicas y Certificación ICONTEC. (2003). Guía Técnica Colombiana GTC93, Guía Para La Ejecución De La Revisión Ambiental Inicial (RAI) y del Análisis De Diferencias (Gap Analysis). Bogotá: el Instituto.

Instituto Colombiano de Normas Técnicas y Certificación ICONTEC. (2004). Sistemas de gestión ambiental. Requisitos con orientación para su uso. NTC-ISO 14001:2004. Bogotá: el Instituto.

López, A. (2003). Globalización y Desarrollo Sostenible: efectos ambientales y sociales de la globalización. En: Revista CIFE. Bogotá: Universidad Santo Tomás, año 6, No 8. ISSN: 0124-3551.

Patiño, M. (1999). Derecho ambiental Colombiano. Incluye código de recursos naturales renovables ley 99 de 1993 y ley del seguro ambiental. Bogotá: LEGIS. ISBN 9586531570. 